EPJ manuscript No.

(will be inserted by the editor)

\title{
Diffractive Final States with the H1 Detector at HERA
}

\author{
Frank-Peter Schilling (for the H1 collaboration) a b \\ DESY, Notkestr. 85, D-22603 Hamburg, Germany \\ Received: date / Revised version: date
}

\begin{abstract}
Recent measurements of diffractive dijet and charm quark production in electron-proton collisions using the $\mathrm{H} 1$ detector at HERA are presented, where the exchanged photon is either almost real or highly virtual. The data are compared with leading and next-to-leading order QCD calculations based on the diffractive parton distributions obtained from a recent DGLAP QCD analysis of H1 inclusive diffractive deep-inelastic scattering data, thus testing QCD factorization in diffractive ep interactions.
\end{abstract}

PACS. $13.60 . \mathrm{Hb}-12.38 . \mathrm{Qk}$

\section{Introduction}

Understanding diffraction in hadronic interactions at high energies, where at least one of the beam hadrons remains intact, losing only a small fraction $x_{\mathbb{P}}$ of its incident longitudinal momentum, represents one of the most important challenges in Quantum Chromodynamics (QCD). The ep collider HERA offers the unique possibility to study hard diffractive processes, such as dijet and heavy quark production, over a wide range of photon virtualities $Q^{2}$.

Recent high precision measurements of the inclusive diffractive deep-inelastic scattering (DIS) cross section by the H1 collaboration [1] have been used to extract diffractive parton distributions (dpdf's) of the proton by means of a DGLAP QCD fit. If QCD factorization is valid in diffractive $e p$ scattering, these dpdf's are universal and can be used to predict cross sections for exclusive hard diffractive processes such as jet and heavy quark production.

The proof of QCD factorization in diffractive $e p$ interactions [2] is restricted to deep-inelastic scattering at large $Q^{2}$ and not valid for the case of an almost real photon (photoproduction). Furthermore, a severe breakdown of factorization has been observed when using dpdf's obtained at HERA to predict diffractive jet production in hadron-hadron interactions at the Tevatron [3].

Here, QCD factorization is tested in DIS to next-toleading order (NLO) by comparing cross section measurements for dijet and $D^{*}$ meson production in diffractive DIS with predictions obtained from convoluting the dpdf's from [1] with NLO order matrix elements for dijet and charm quark production [4]. In addition, diffractive dijet photoproduction data are compared with leading order (LO) Monte Carlo predictions [5].

\footnotetext{
${ }^{a}$ e-mail: fpschill@mail.desy.de

b Talk presented at the Intl. Europhysics Conference on High Energy Physics EPS 2003, Aachen, July 2003
}

\subsection{Kinematics}

Fig. 1 shows an example diagram for diffractive dijet or charm quark production at HERA.

The photon (virtual-

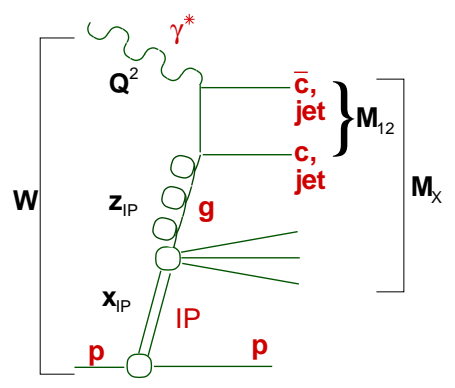

Fig. 1. Diagram for diffractive jet or charm production at HERA.

photon-proton centre-of-mass encre ass energy is labelled $W$ and the invariant masses of the diffractively produced system and of the two partons emerging from the hard sub-process are denoted $M_{X}$ and $M_{12}$, respectively. The inelasticity variable $y$ is given by $y s=W^{2}+Q^{2}$, where $s$ is the squared ep centre-of-mass energy.

\subsection{H1 Diffractive Parton Distributions}

QCD factorization permits to express the diffractive DIS $\gamma^{*} p$ cross section as a convolution of diffractive parton distributions $p_{i}^{D}$ and partonic cross sections $\hat{\sigma}^{\gamma^{*} i}$ :

$$
\frac{\mathrm{d}^{2} \sigma_{\gamma^{*} p}^{D}}{\mathrm{~d} x_{\mathbb{P}} \mathrm{d} t} \sim \sum_{i} \hat{\sigma}^{\gamma^{*} i}\left(x, Q^{2}\right) \otimes p_{i}^{D}\left(x, Q^{2}, x_{\mathbb{P}}, t\right)
$$


where $t$ is the squared 4-momentum transferred at the proton vertex. The dpdf's are universal and obey the DGLAP evolution equations. In the QCD fit to the $\mathrm{H} 1$ inclusive diffractive DIS data in [1], Regge factorization was assumed as well (consistent with the data within the present level of precision), i.e.

$$
p_{i}^{D}\left(x, Q^{2}, x_{\mathbb{P}}, t\right)=f_{\mathbb{P}}\left(x_{\mathbb{P}}, t\right) p_{i}^{\mathbb{P}}\left(z=x / x_{\mathbb{P}}, Q^{2}\right),
$$

so that the shape of the dpdf's is independent of $x_{\mathbb{P}}, t$ and the normalization is controlled by a flux factor $f_{\mathbb{P}}$, for which a value for the pomeron intercept $\alpha_{\mathbb{P}}(0)=1.173$ is used. The parton distributions $p_{i}^{\mathbb{P}}$, as determined from both the LO and NLO QCD fits, are dominated by the gluon density and extend to large fractional momenta $z$.

\section{Dijet Production in Diffractive DIS}

To test QCD factorization for diffractive dijet production in DIS, the H1 cross sections from [6] were used, corresponding to the kinematic range $4<Q^{2}<80 \mathrm{GeV}^{2}$ and $x_{\mathbb{P}}<0.05$. The cross sections were corrected to asymmetric cuts on the jet transverse momentum $p_{T, 1(2)}>$ $5(4) \mathrm{GeV}$, to facilitate comparisons with NLO calculations.

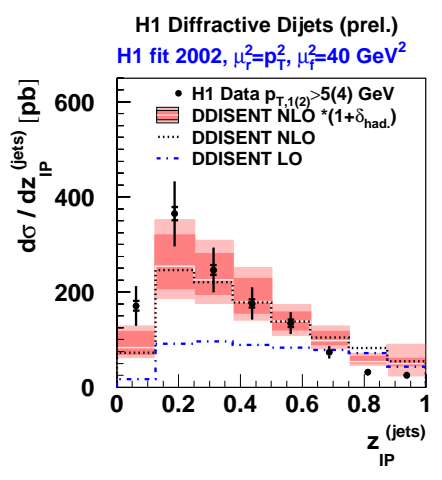

Fig. 2. Diffractive DIS dijet cross section as a function of $z_{\mathbb{P}}^{(j e t s)}$, an estimator for the parton momentum fraction of the diffractive exchange entering the hard subprocess.

The NLO dpdf's obtained in [1] were convoluted with NLO matrix elements for dijet production by means of the DISENT [7] program, as suggested in [8]. The renormalization and factorization scales were set to the average $p_{T}$ of the two highest $p_{T}$ partons. $\alpha_{s}$ was set via $\Lambda_{Q C D}^{n=4}=200 \mathrm{MeV}$, as in the QCD fit. To allow comparisons with the measured data, the same jet algorithm as for the data, as well as hadronization corrections, were applied.

Comparisons of the LO and NLO QCD calculations with the dijet data are shown in Figs. 2 and 3. The size of the NLO corrections is on average more than a factor 2 (increasing with decreasing $p_{T}$ and $Q^{2}$ ). The inner error band of the NLO calculations represents the renormalization scale uncertainty, whereas the outer band includes the uncertainty in the hadronization corrections. Within the uncertainties, the data are well described in both shape and normalization by the NLO calculations based on the dpdf's from [1], in agreement with QCD factorization.
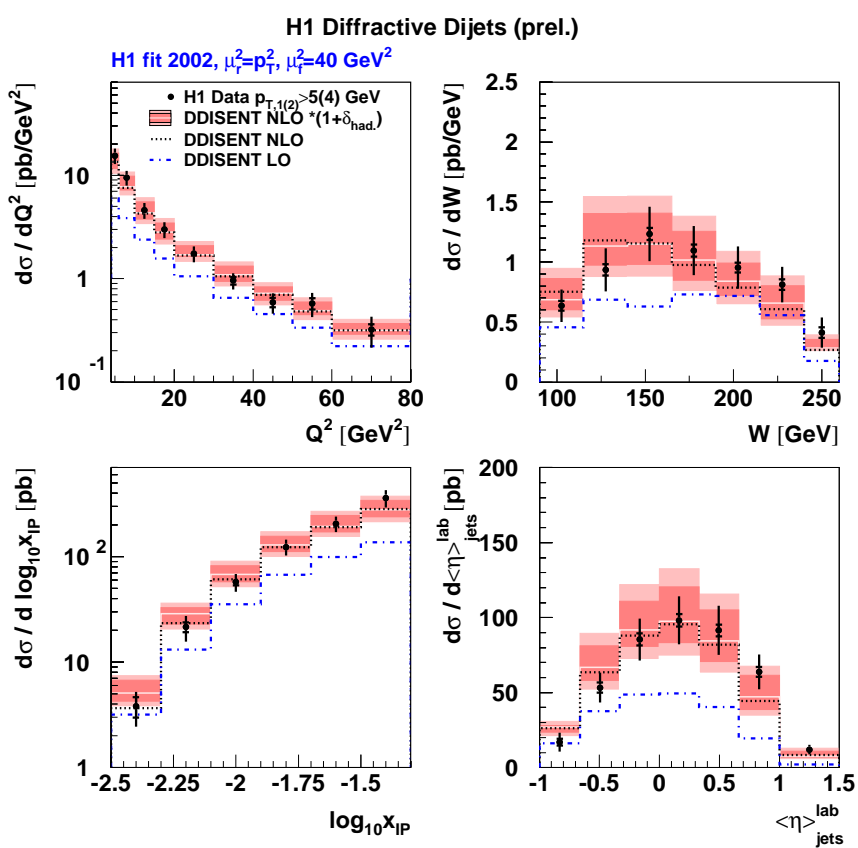

Fig. 3. Diffractive DIS dijet cross sections differential in $Q^{2}$, $W, \log _{10} x_{\mathbb{P}}$ and the average dijet pseudorapidity $\langle\eta\rangle_{\text {jets }}^{l a b}$.
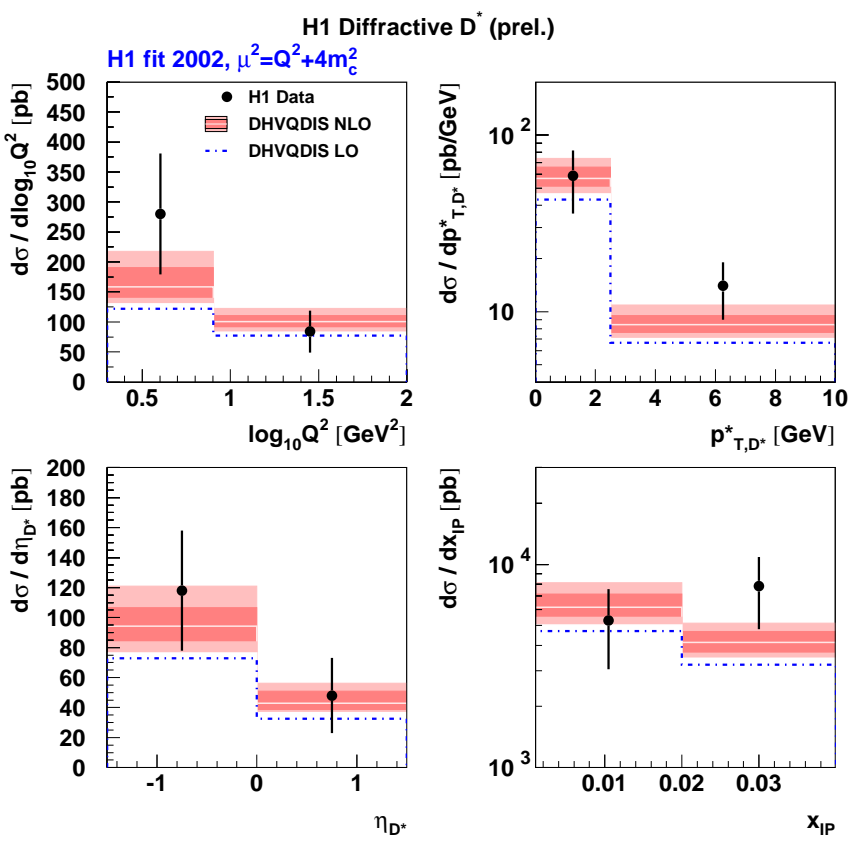

Fig. 4. Diffractive DIS $D^{*}$ meson cross sections differential in $Q^{2}, p_{T, D^{*}}^{*}, \eta_{D^{*}}$ and $x_{\mathbb{P}}$.

\section{$3 D^{*}$ Meson Production in Diffractive DIS}

In [9], cross sections for $D^{*}$ meson production in diffractive DIS were published for the kinematic range $2<Q^{2}<$ $100 \mathrm{GeV}^{2}, x_{\mathbb{P}}<0.04$ and $p_{T, D^{*}}^{*}>2 \mathrm{GeV}$, where the latter variable corresponds to the transverse momentum of the $D^{*}$ meson in the photon-proton centre-of-mass frame. 

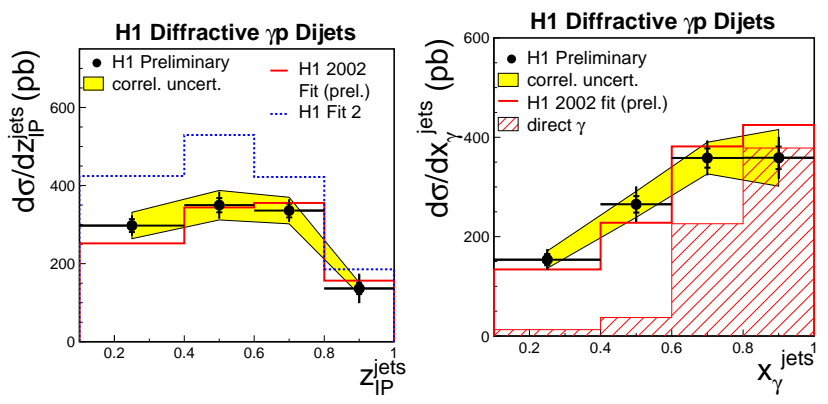

Fig. 5. Diffractive dijet photoproduction cross sections differential in $z_{\mathbb{P}}^{\text {jets }}$ and $x_{\gamma}^{\text {jets }}$.

Both LO and NLO QCD calculations were performed using the diffractive version [10] of HVQDIS [11], interfaced to the H1 dpdf's. The renormalization and factorization scales were set to $\mu^{2}=Q^{2}+4 m_{c}^{2}$. Further parameter values are a charm quark mass of $m_{c}=1.5 \mathrm{GeV}$, a $c \rightarrow D^{*}$ hadronization fraction of $f\left(c \rightarrow D^{*}\right)=0.233$ and $\epsilon=0.078$ for the used Peterson fragmentation function.

A comparison of the calculations with the $D^{*}$ data is shown in Fig. 4. The inner error band of the NLO calculation represents the renormalization scale uncertainty, whereas the outer error band includes variations of $m_{c}$ and $\epsilon$. Within the uncertainties, the data are well described in both shape and normalization by the NLO calculations, using the dpdf's from [1], supporting the idea of QCD factorization.

\section{Dijets in Diffractive Photoproduction}

In [5], H1 has presented a measurement of diffractive dijet photoproduction, i.e. for $Q^{2} \sim 0$. The cross sections correspond to the kinematic range $Q^{2}<0.01 \mathrm{GeV}^{2}, 0.3<$ $y<0.65, x_{\mathbb{P}}<0.03$ and $p_{T, 1(2)}>5(4) \mathrm{GeV}$, where jets are defined using the $k_{T}$ algorithm.

The data are compared in Figs. 5 (absolute cross sections) and 6 (normalized cross sections) with LO Monte Carlo predictions using both the LO version of the dpdf's from [1], as well as an earlier version from [12]. $x_{\gamma}^{\text {jets }}$ is an estimator for the momentum fraction of the photon entering the hard process, which is used to distinguish between direct and resolved photon interactions, where in the latter case the photon develops hadronic structure, and a parton from the photon with momentum fraction $x_{\gamma}$ enters the hard process.

For both direct and resolved interactions, the dijet data are well described by the Monte Carlo predictions if the new H1 dpdf's from [1] are used. Thus, the data are not consistent with a breakdown of factorization even in resolved photoproduction, which resembles hadron-hadron scattering where the presence of the second hadron may lead to a suppression of the rate of diffractive events due to spectator interactions.

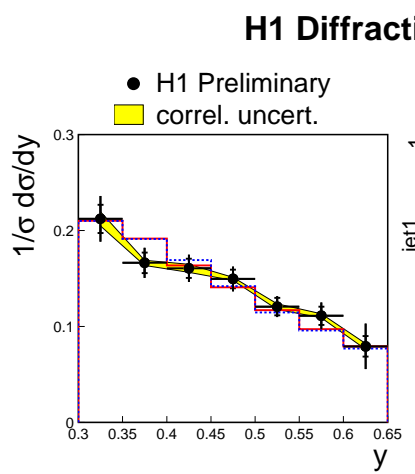

(a)

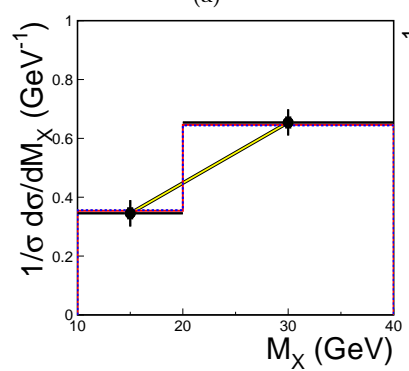

(c)

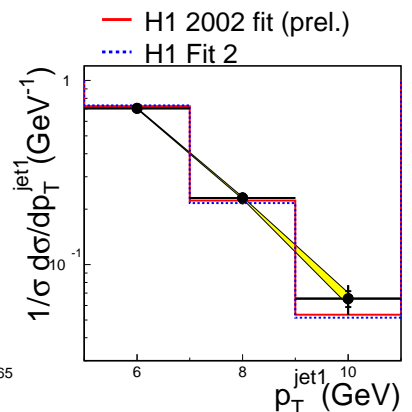

(b)

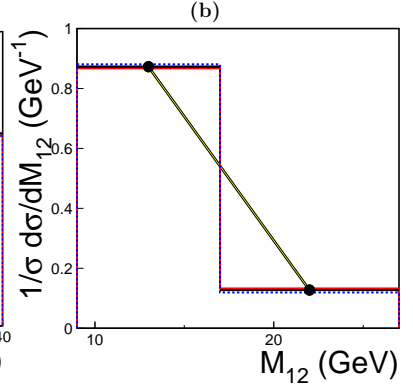

(d)

Fig. 6. Normalized diffractive dijet photoproduction cross sections differential in $y, p_{T}^{\text {jet } 1}, M_{X}$ and $M_{12}$.

\section{Conclusions}

The new H1 diffractive parton distributions have been interfaced with NLO QCD calculations and compared with dijet and $D^{*}$ production data in diffractive DIS. The good agreement within the uncertainties lends support for the validity of QCD factorization in diffractive DIS, now tested to next-to-leading order. In addition, also photoproduction diffractive dijet measurements are described in shape and normalization by Monte Carlo predictions using these dpdf's, not indicating any breakdown of factorization in diffractive $e p$ interactions at HERA.

\section{References}

1. H1 Coll., paper 980 subm. to ICHEP 2002, Amsterdam.

2. J. Collins, Phys. Rev. D 57, (1998) 3051 [erratum-ibid. D 61, (2000) 019902].

3. CDF Coll., T. Affolder et al., Phys. Rev. Lett. 84, (2000) 5043.

4. H1 Coll., paper 113 subm. to EPS 2003, Aachen.

5. H1 Coll., paper 087 subm. to EPS 2003, Aachen.

6. H1 Coll., C. Adloff et al., Eur. Phys. J. C 20, (2001) 29.

7. S. Catani, M. H. Seymour, Nucl. Phys. B 485, (1997) 29 [erratum-ibid. B510, (1997) 503].

8. F. Hautmann, JHEP 0210, (2002) 025.

9. H1 Coll., C. Adloff et al., Phys. Lett. B 520, (2001) 191.

10. L. Alvero, J. C. Collins, J. J. Whitmore, "Tests of factorization in diffractive charm production and double pomeron exchange", hep-ph/9806340.

11. B. W. Harris, J.Smith, Phys. Rev. D 57, (1998) 2806.

12. H1 Coll., C. Adloff et al., Z. Phys. C 76, (1997) 613. 